$\Rightarrow$ LIGHT-MATTER INTERACTIONS

\title{
Longer times and stronger signals
}

Electron-photon coupling makes it possible to image nanostructures on ultrafast timescales. The technique works even better with free electrons than with those bound within atoms, because they can interact at higher energy scales and shorter timescales. However, the use of free electrons has so far been limited by their weak coupling to photons. Now, two papers published in Nature report stronger coupling between photons and free electrons.
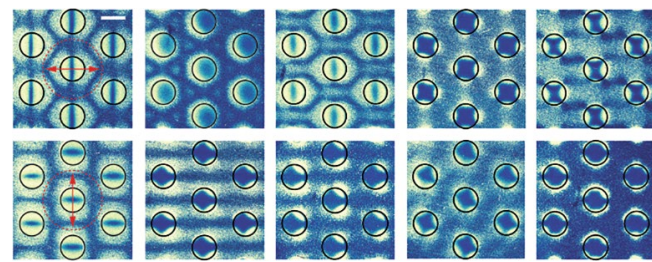

Credit: Adapted from Wang, K. et al. Springer Nature Limited
In one paper, Kangpeng Wang and colleagues report coupling of free electrons to photonic cavities. The Bloch modes - periodic oscillations of photons within the cavity - were directly imaged by the electron beam (pictured) and the energy, momentum and polarization spectra of the cavity modes were also measured. The electron energy-loss spectrum was used to calculate the lifetime of the cavity mode. The high-Q cavity showed a much longer lifetime than the low-Q cavity, indicating that the quality of the cavity could be used to enhance the strength of the electron-photon interaction. Stronger interactions mean that the energy of the incoming electron beam can be low, so as not to damage sensitive structures, while still providing high resolution images.

In the other paper, Ofer Kfir and co-workers increase the electron-photon interaction strength by coupling the electrons to whispering gallery modes modes formed by confining light within dielectric resonators using total internal reflection. Unlike ordinary modes, which are localized in space and so only affect electrons for a short time while they pass through, these whispering gallery modes can interact with travelling electrons. Thus many more photons are able to interact with the electron beam, enhancing the overall strength of the interaction.

Ankita Anirban

ORIGINAL ARTICLES Wang, K. et al. Coherent interaction between free electrons and a photonic cavity. Nature https://doi.org/10.1038/s41586020-2321-x (2020) | Kfir, O. et al. Controlling free electrons with optical whispering-gallery modes. Nature, https://doi.org/10.1038/s41586-020-2320-y (2020) 